\title{
Artigo/Article
}

\section{Distribuição espacial e características dos criadouros de Aedes albopictus e Aedes aegypti em Fortaleza, Estado do Ceará}

\author{
Spatial distribution and breeding site characteristics of Aedes albopictus and Aedes aegypti \\ in Fortaleza, State of Ceará
}

\author{
Victor Emanuel Pessoa Martins ${ }^{1}$, Carlos Henrique Morais de Alencar ${ }^{2}$, Patrícia Emília Gomes Facó ${ }^{3}$, \\ Rosa Fireman Dutra ${ }^{3}$, Carlucio Roberto Alves ${ }^{3}$, Ricardo José Soares Pontes ${ }^{2}$ e Maria Izabel Florindo Guedes ${ }^{1}$
}

\begin{abstract}
RESUMO
Introdução: Desde seu registro, em 2005, no município de Fortaleza, o Aedes albopictus tem exibido uma rápida dispersão. Um estudo visando à identificação das áreas de sua ocorrência, os seus criadouros e a associação com Aedes aegypti e outros culicídeos foi realizado durante o ano de 2008. Métodos: Foram coletadas, de janeiro a julho de 2008, amostras de formas imaturas de culicídeos provenientes de imóveis situados nos bairros de Fortaleza, ressaltando-se algumas características dos criadouros, tais como localização (intradomicílio ou peridomicílio), presença de cobertura (proteção contra a incidência de raios solares e chuva), turbidez da água (água límpida e não límpida), material constituinte, volume, altura em relação ao nível do solo e presença simultânea de diferentes espécies de culicídeos no mesmo criadouro. Resultados: A localização no intradomicílio foi um fator importante para os criadouros do Aedes albopictus [RP=0,52 IC95\% (0,33-0,81)], por outro lado, a turbidez da água e a cobertura do depósito não se mostraram como diferenciadores para a infestação [p>0,05]. Para o Aedes aegypti a ausência de turbidez da água foi significativa para a infestação nos criadouros [RP=1,14 IC95\% (1,06-1,22)]. Conclusões: a ausência de uma das espécies nos criadouros favorecia sua infestação por outra; criadouros não infestados por Aedes albopictus tinham uma prevalência de infestação de 2,05 [IC95\%1,72-2,44] vezes maior pelo Aedes aegypti. Não houve associação significativa entre volume e altura do criadouro e infestação por ambas as espécies. As duas espécies encontram-se dispersas por todo o município, ocupando os mais diversos tipos de criadouros. No entanto, pode-se identificar uma ligeira separação física, com uma infestação maior do Aedes albopictus no peridomicílio.
\end{abstract}

Palavras-chaves: Aedes aegypti. Aedes albopictus. Distribuição espacial. Características dos criadouros.

\begin{abstract}
Introduction: Since the registration of Aedes albopictus in the municipality of Fortaleza in 2005, it has shown rapid dispersion. A study aiming to identify its occurrence areas, breeding sites and associations with Aedes aegypti and other culicids was carried out during 2008. Methods: Between January and July 2008, samples of immature forms of culicids were gathered from properties located in districts of Fortaleza. Certain features of the breeding sites were highlighted, such as location (indoors or outdoors), presence of covering (protection against impact of sunlight and rain), water turbidity (water clear or not clear), constituent material, volume, height above ground level and simultaneous presence of different species of culicids in the same breeding site. Results: Indoor location was an important factor for the breeding sites for Aedes albopictus [PR $=0.52 ; 95 \%$ CI: 0.33-0.81]. On the other hand, water turbidity and reservoir covering were not shown to be differentiators regarding infestation $[\mathrm{p}>0.05]$. Absence of water turbidity was significant for Aedes aegypti infestation in breeding sites $[\mathrm{PR}=1.14$; 95\% CI: 1.06-1.22]. Conclusions: Absence of one of the species from the breeding sites enhanced the infestation by another species. Breeding sites that had not been infested by Aedes albopictus had a prevalence of infestation by Aedes aegypti that was 2.05 times greater [95\% CI: 1.72-2.44]. There was no significant association between volume and height of the breeding sites and infestation by both species. Both species were found to be dispersed throughout the municipality, occupying a wide diversity of breeding sites. However, a slight physical separation could be identified, with higher infestation with Aedes albopictus outdoors.
\end{abstract}

Key-words: Aedes aegypti. Aedes albopictus. Spatial distribution. Breeding site characteristics.

1. Rede Nordeste de Biotecnologia/RENORBIO/UECE, Laboratório de Bioquímica Humana, Universidade Estadual do Ceará, Fortaleza, CE. 2. Programa de Pós-Graduação em Saúde Coletiva, Departamento de Saúde Comunitária, Faculdade de Medicina, Universidade Federal do Ceará, Fortaleza, CE. 3. Laboratório de Bioquímica Humana, Universidade Estadual do Ceará, Fortaleza, CE.

Endereço para correspondência: Dr. Victor Emanuel Pessoa Martins. Laboratório de Bioquímica Humana/ UECE. Av. Paranjana 1700, Campus do Itaperi, Bloco D/1º andar, 60740-000 Fortaleza, CE.

Tel: 5585 3101-9822

e-mail: victorpessoabiologo@yahoo.com.br

Recebido para publicação em 31/05/2009

Aceito em 07/12/2009

\section{INTRODUÇÃO}

Reconhecido vetor dos vírus dengue e da febre amarela urbana, o Aedes aegypti foi considerado uma espécie erradicada no Brasil em 1955. No entanto, no final dos anos 1960 registra-se sua reintrodução no país, facilitada pelas fronteiras estabelecidas entre os estados da Região Norte e países como a Venezuela e as Guianas, onde essa espécie ainda não havia sido erradicada. A partir de então, tem sido responsável pela transmissão dos vírus dengue em epidemias registradas no território brasileiro desde o início dos anos $1980^{1}$. Atualmente, o Aedes aegypti encontra-se disseminado em todos os Estados da Federação, onde suas populações apresentam elevada susceptibilidade à infecção natural pelos vírus dengue e da febre amarela ${ }^{2,3}$. Um segundo vetor em potencial mostra-se presente no Brasil desde 1986, o Aedes albopictus, que, até 2003, não havia sido registrado em apenas sete dos Estados brasileiros $^{4,5}$. Trata-se de uma espécie que atua como vetor secundário dos vírus dengue em zonas rurais $\mathrm{e}$ urbanas. Porém, no Brasil, ainda não foi incriminado em surtos ou epidemias da doença, mas que, sob condições experimentais, mostra-se competente à infecção e à transmissão de 22 outros arbovírus ${ }^{6,7}$.

O Estado do Ceará, em especial o município de Fortaleza, desde o registro dos primeiros casos de dengue, na década de 1980, vem enfrentando grandes dificuldades em lidar com as epidemias da doença, as quais exigem a adoção de medidas que visam ao seu controle, compreensão de seus mecanismos efetores e veículos de transmissão. Desde então, o combate ao Aedes aegypti e ao Aedes albopictus, este tendo sido registrado em território cearense em $2005^{8}$, tem sido prioridade nas ações do Programa de Controle da Dengue. Embora essas duas espécies compartilhem vários aspectos no tocante à biologia $\mathrm{e}$ ao comportamento ${ }^{7}$, ressaltam-se algumas diferenças no que diz respeito à sua frequência nos mais variados ambientes alterados pela ação humana, os quais podem influenciar, sobremaneira, na competência vetorial de suas populações no Brasil ${ }^{9}$. 
Este trabalho objetiva identificar as áreas de ocorrência do Aedes albopictus no município de Fortaleza, assim como caracterizar os tipos de criadouros nos domicílios que favorecem sua manutenção e relacioná-los à ocorrência concomitante de Aedes aegypti e de outros culicídeos.

\section{MÉTODOS}

O Município de Fortaleza, capital do Estado do Ceará, está localizado no litoral norte da região Nordeste do Brasil. Com $313,14 \mathrm{~km}^{2}$ de área e com densidade demográfica de 7.851,27 habitantes $/ \mathrm{km}^{2}$, é atualmente a quarta maior capital do país em termos populacionais, estimando-se sua população residente total, no ano de 2008, em 2.458.545 pessoas $^{10}$.

Está organizado administrativamente em seis Secretarias Executivas Regionais (SER) desde 1997, onde estão seus 116 bairros.

Em consonância com as atividades referentes ao Programa de Controle da Dengue em Fortaleza, realizadas pela Secretaria Municipal de Saúde, foram coletadas, no período de janeiro a julho de 2008, amostras de formas imaturas de culicídeos provenientes de imóveis situados nos bairros de Fortaleza. As pesquisas realizadas nas áreas urbanas cobriram residências, terrenos baldios, comércios e outros possíveis pontos para o desenvolvimento larvário de mosquitos.

Os espécimes coletados foram acondicionados em tubos de vidro de $10 \mathrm{ml}$, contendo água do próprio criadouro, devidamente identificados, com etiquetas nas quais ressaltavam-se algumas características dos criadouros, tais como: localização (intradomicílio ou peridomicílio), presença de cobertura (proteção contra a incidência de raios solares e chuva), turbidez da água (água límpida e não límpida), material constituinte, volume, altura em relação ao nível do solo e presença simultânea de diferentes espécies de culicídeos no mesmo criadouro. Em seguida, as amostras larvárias foram enviadas ao Laboratório de Bioquímica Humana, da Universidade Estadual do Ceará, onde foram transferidas para recipientes plásticos de $200 \mathrm{ml}$, permanecendo nestes até atingirem o estágio de pupa. Durante este período, as larvas foram alimentadas com ração à base de soja. A partir de então, as pupas foram transferidas para recipientes plásticos de $500 \mathrm{ml}$, adaptados para a manutenção das formas aladas emergentes. Uma vez atingido o estágio adulto, os mosquitos foram mantidos em temperatura de $4^{\circ} \mathrm{C}$, durante $5 \mathrm{~min}$, com o propósito de lhes produzir um efeito anestésico, facilitando sua posterior identificação. Todas as amostras coletadas foram identificadas quanto à presença de Aedes aegypti, Aedes albopictus e Culex spp, mediante chave de classificação específica ${ }^{11}$.

A análise dos dados foi realizada utilizando-se o programa Epi info 6.04d, observando-se um intervalo de confiança de $95 \%$, e testes de chi quadrado e Mann-Whitney foram utilizados para medir as diferenças nas diversas proporções estudadas e comparação das medianas, respectivamente.

\section{RESULTADOS}

Foram coletadas 1.391 amostras procedentes dos mais diversos bairros de Fortaleza, dos quais 78 (67,2\%) apresentaram criadouros para o Aedes aegypti e 82 (70,7\%) para o Aedes albopictus (Figuras 1 e 2).

Observa-se, a partir da análise da Figura 1, a ocorrência de criadouros para o Aedes aegypti em quase todo o município de Fortaleza, tendo-se como áreas de maior predominância as referentes

Distribuição de imóveis positivos por bairro Nenhum registro de 1 a 10 imóveis positivos de 11 a 20 imóveis positivos de 21 a 30 imóveis positivos de 31 a 40 imóveis positivos Mais de 40 imóveis positivos

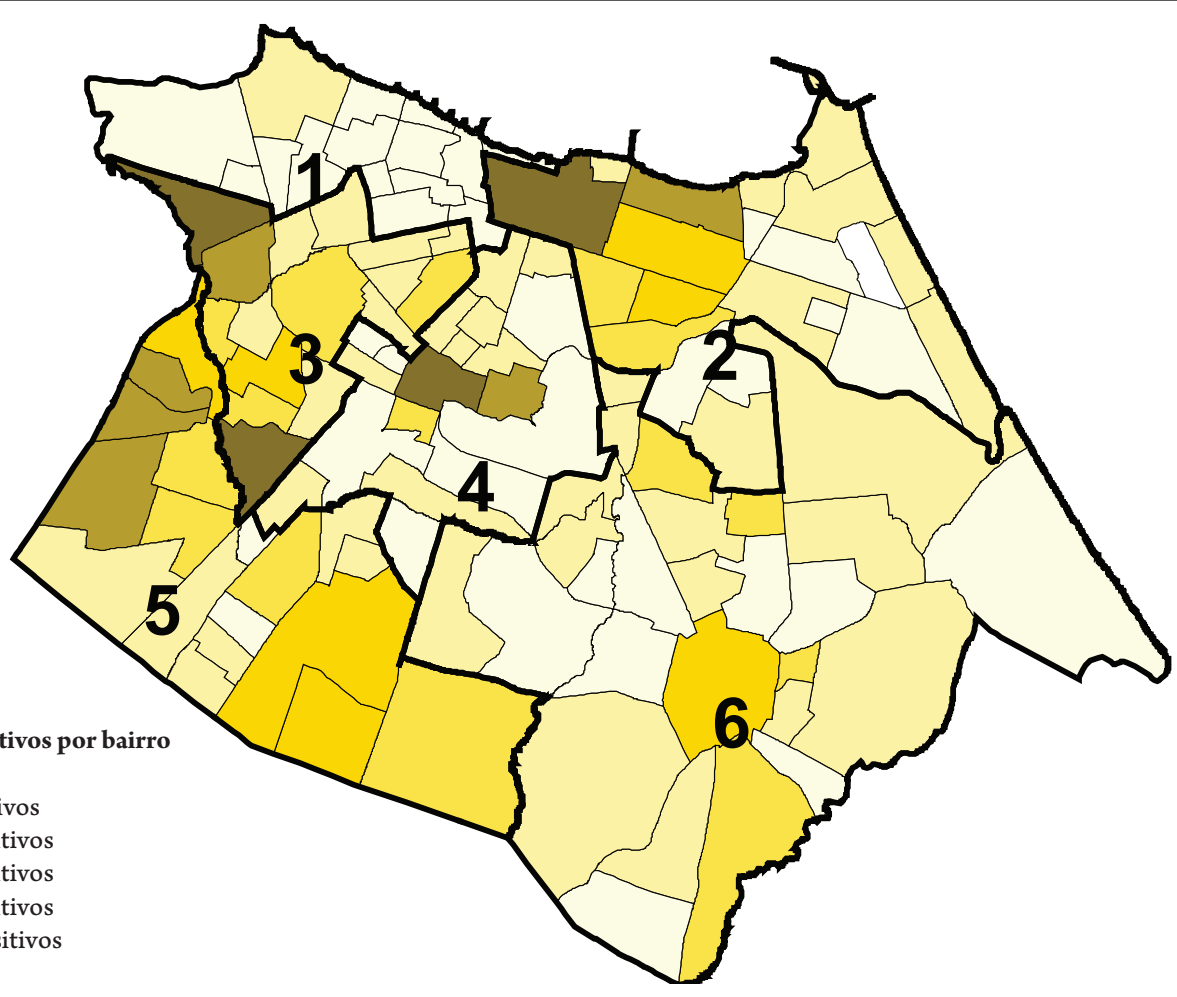




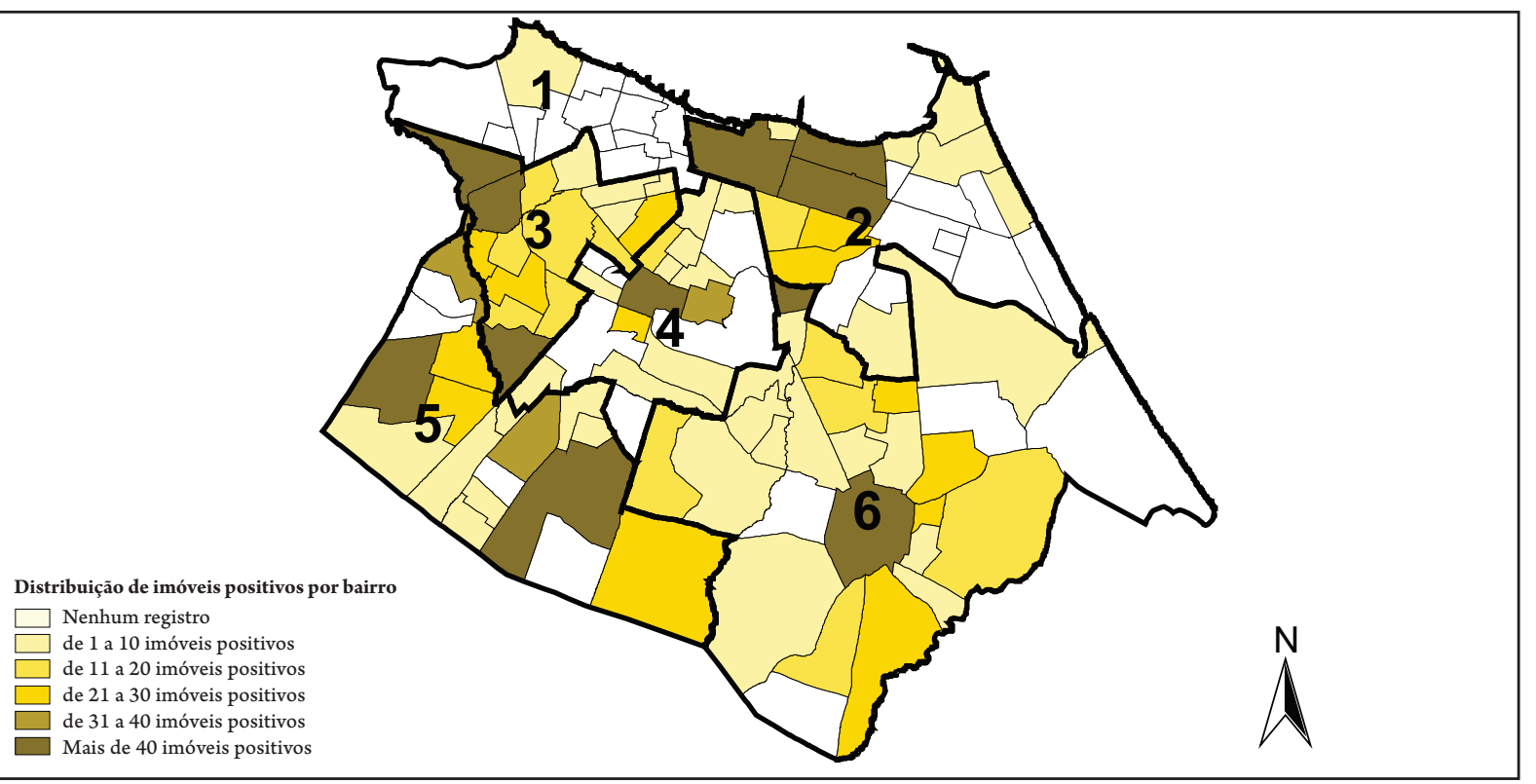

FIGURA 2 - Bairros com presença de criadouros de Aedes albopictus, no período de janeiro a julho de 2008, Fortaleza, CE.

às SER 3 e 5. Da mesma forma, observa-se uma ampla distribuição dos criadouros para o Aedes albopictus, e também significativa concentração nas SER 3 e 5, mas também na SER 6, como mostrado na Figura 2.

Quanto aos tipos de depósitos mais frequentados pelas espécies em estudo, para Aedes aegypti destacam-se os tanques (13,8\%), os tambores $(11,8 \%)$ e as caixas d'água $(9,8 \%)$, enquanto para Aedes albopictus, as caixas d'água (11,6\%), os tanques $(10,5 \%)$ e os potes $(9,4 \%)$ foram os criadouros mais frequentados.

Em relação ao material constituinte dos criadouros utilizados por ambas as espécies, há uma preferência pelos depósitos de alvenaria (33,9\% pelo Aedes aegypti e 32,8\% pelo Aedes albopictus) e de plástico (35,6\% pelo Aedes aegypti e 32,8\% pelo Aedes albopictus), sendo os de barro (11,4\%) também bastante frequentados pelo Aedes aegypti e os de metal (14,5\%) pelo Aedes albopictus.

Observa-se que na Tabela 1, a ausência de turbidez da água foi significativa para a infestação dos criadouros por Aedes aegypti, enquanto que a presença de cobertura e sua localização no imóvel não foram significativas. Com relação ao criadouro do Aedes albopictus, observa-se que a sua localização no peridomicílio é fator que aumenta o risco de infestação por esta espécie. Por outro lado, a turbidez da água e a presença de cobertura dos criadouros não demonstraram influência em sua infestação.

Verificou-se, ainda, que nos criadouros onde houve a presença de Aedes aegypti não foram encontrados números significativos de espécimes de Aedes albopictus e Culex spp, o mesmo sendo observado nos criadouros de Aedes albopictus (Tabela 1).

Observa-se, de acordo com a Tabela 2, que o volume dos criadouros infestados por Aedes aegypti têm uma mediana superior àqueles infestados pelo Aedes albopictus, o mesmo podendo ser observado em relação à sua amplitude. Esta última espécie apresenta a possibilidade de colonizar criadouros desde $100 \mathrm{ml}$ até volumes da ordem de 8.000L (Tabela 2).

Com relação à altura dos criadouros, a mediana daqueles infestados pelo Aedes aegypti foi bem superior a dos infestados por Aedes albopictus. Apesar da grande amplitude observada para os criadouros infestados por esta última espécie, aproximadamente $90 \%$ destes encontram-se em alturas inferiores a $1 \mathrm{~m}$ (Tabela 2).
TABELA 1 - Características dos criadouros em domicílios infestados por Aedes aegypti e Aedes albopictus, no município de Fortaleza, no período de janeiro a julho de 2008.

\begin{tabular}{|c|c|c|c|c|c|c|}
\hline \multirow[b]{2}{*}{ Variável } & \multirow[b]{2}{*}{ Total } & \multicolumn{2}{|c|}{ Infestaçãc } & \multicolumn{2}{|c|}{ Razão de infestação } & \multirow[b]{2}{*}{ Valor-p } \\
\hline & & Positivos & $(\%)$ & pontual & $\mathrm{IC} 95 \%$ & \\
\hline \multicolumn{7}{|l|}{ Aedes aegypti } \\
\hline \multicolumn{7}{|l|}{ Localização } \\
\hline no peridomicílio & 708 & 546 & 77,1 & 1 & - & \\
\hline no intradomicílio & 293 & 233 & 79,5 & 1,03 & $0,96-1,10$ & $0 \quad 0,405$ \\
\hline \multicolumn{7}{|l|}{ Cobertura } \\
\hline sem cobertura & 591 & 474 & 80,2 & 1 & - & \\
\hline com cobertura & 182 & 154 & 84,6 & 1,05 & $0,97-1,13$ & 30,182 \\
\hline \multicolumn{7}{|l|}{ Turbidez da água } \\
\hline água não límpida & 407 & 305 & 74,9 & 1 & - & \\
\hline água límpida & 426 & 365 & 85,7 & 1,14 & $1,06-1,22$ & 20,000 \\
\hline \multicolumn{7}{|c|}{ Presença de Aedes albopictu } \\
\hline $\operatorname{sim}$ & 193 & 77 & 39,9 & 1 & - & \\
\hline não & 1.197 & 980 & 81,9 & 2,05 & $1,72-2,44$ & $4 \quad 0,000$ \\
\hline \multicolumn{7}{|l|}{ Presença de Culex spp } \\
\hline $\operatorname{sim}$ & 267 & 43 & 16,1 & 1 & - & \\
\hline não & 1.123 & 1015 & 90,4 & 5,61 & $4,26-7,38$ & $8 \quad 0,000$ \\
\hline
\end{tabular}

\section{Aedes albopictus}

Localização

no peridomicílio

no intradomicílio

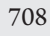

$101 \quad 14,3$

obertura

sem cobertura

com cobertura

$101 \quad 14,3$

urbidez da Água água não límpida água límpida

Presença de Aedes aegypti

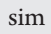

1

182

$20 \quad 10,9$

$0,80 \quad 0,50-1,27 \quad 0,341$

não

$\begin{array}{llll}407 & 54 & 13,3 & 1\end{array}$

$\begin{array}{llllll}426 & 48 & 11,3 & 0,84 & 0,58-1,22 & 0,378\end{array}$

Presença de Culex spp

\begin{tabular}{|c|c|c|c|c|c|}
\hline $\operatorname{sim}$ & 267 & 13 & 4,9 & 1 & - \\
\hline não & 1.123 & 180 & 16,0 & 0,30 & $0,17-0,52$ \\
\hline
\end{tabular}

IC: intervalo confiança. 
TABELA 2 - Características dos criadouros quanto aos aspectos de volume e altura, presentes em imóveis localizados em bairros infestados por Aedes aegypti e Aedes albopictus, no período de janeiro a julho de 2008, Fortaleza, CE.

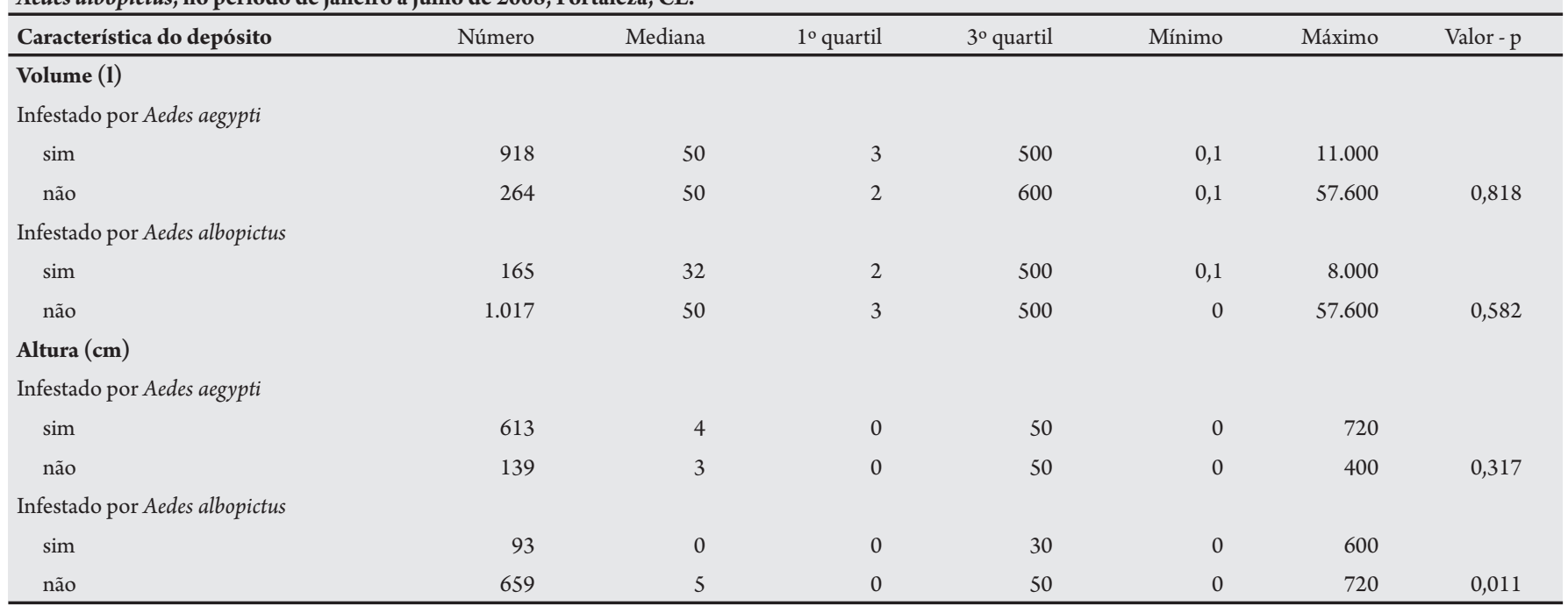

TABELA 3 - Distribuição de formas imaturas de Aedes aegypti e Aedes albopictus, categorizada por volume e altura dos criadouros, no período de janeiro a julho de 2008, Fortaleza, CE.

\begin{tabular}{|c|c|c|c|c|c|}
\hline \multirow[b]{2}{*}{ Variável } & \multirow[b]{2}{*}{ Total } & \multirow{2}{*}{$\begin{array}{c}\text { Infestação } \\
(\%)\end{array}$} & \multicolumn{2}{|c|}{ Razão de infestação } & \multirow[b]{2}{*}{ Valor-p } \\
\hline & & & pontual & IC95\% & \\
\hline \multicolumn{6}{|c|}{ Aedes aegypti } \\
\hline \multicolumn{6}{|c|}{ Volume (ml) } \\
\hline$\leq 50$ & 637 & 77,7 & 1 & - & \\
\hline$\geq 50$ & 545 & 77,6 & 0,99 & $0,93-1,06$ & 0,969 \\
\hline Altura (m) & 668 & & & & \\
\hline$\leq 1$ & 81,4 & 1 & - & & \\
\hline$\geq 1$ & 84 & 82,1 & 1,00 & $0,90-1,12$ & 0,875 \\
\hline \multicolumn{6}{|c|}{ Aedes albopictus } \\
\hline \multicolumn{6}{|c|}{ Volume (ml) } \\
\hline$\leq 32$ & 573 & 14,5 & 1 & - & \\
\hline$\geq 32$ & 817 & 13,5 & 0,92 & $0,71-1,21$ & 0,587 \\
\hline \multicolumn{6}{|l|}{ Altura (m) } \\
\hline$\leq 1$ & 668 & 12,7 & 1 & - & \\
\hline$\geq 1$ & 84 & 9,5 & 0,74 & $0,37-1,48$ & 0,401 \\
\hline
\end{tabular}

IC: intervalo confiança.

Dividindo-se o volume dos criadouros pela mediana, observase que não houve diferenças significativas em relação à infestação tanto pelo Aedes aegypti quanto pelo Aedes albopictus. O mesmo foi observado em relação a depósitos com altura até $1 \mathrm{~m}$ acima do solo, em relação àqueles mais altos (Tabela 3 ).

\section{DISCUSSÃO}

Desde sua introdução no Brasil, o Aedes aegypti tem sido largamente combatido, uma vez que, além dos vírus dengue, está relacionado com a transmissão da febre amarela urbana. Sua elevada antropofilia, sendo encontrado facilmente em áreas densamente povoadas, permitiu a sua rápida disseminação pelo território nacional ${ }^{11}$. Por outro lado, o Aedes albopictus ainda não foi incriminado pela transmissão dos vírus dengue no Brasil, embora cepas desses vírus tenham sido isoladas de formas imaturas desta espécie $^{12}$. É bastante eclético quanto aos tipos de criadouros que costuma frequentar, assim como tem apresentado uma significativa adaptação ao ambiente urbano, fato importante no que concerne à sua potencialidade em participar do ciclo de transmissão dos vírus dengue nesse ambiente.

De uma maneira geral, aceita-se a teoria de que o Aedes albopictus foi originalmente uma espécie selvagem que procriava e alimentavase nas margens das florestas, passando a adaptar-se ao domicílio e ao peridomicílio dos imóveis nas diversas áreas de sua distribuição ${ }^{13,14}$. Nos últimos anos, o ambiente urbano, cada vez mais, tem oferecido condições para sua permanência, no qual, inclusive, é possível observar uma forte tendência de sobreposição de nichos em relação ao Aedes aegypti.

O Aedes albopictus pode utilizar os mesmos tipos de criadouros artificiais que o Aedes aegypti $i^{15}$. No entanto, neste estudo, observouse que em criadouros onde foram encontradas formas imaturas de Aedes albopictus havia uma baixa prevalência de formas imaturas de Aedes aegypti, o que sugere a existência de uma competição entre essas espécies por recursos do meio, obrigando-as, neste caso, a elaborar novas estratégias de adaptação, com fins de minimizar os efeitos dessa provável competição. Por outro lado, na região do Vale do Paraíba (SP), observaram-se associações interespecíficas de Aedes albopictus com espécies autóctones, onde fora encontrado coabitando com espécimes de Aedes terrens e Culex quinquefasciatus ${ }^{16}$.

A localização dos criadouros no peridomicílio revelou uma forte associação com a infestação pelo Aedes albopictus, corroborando os resultados obtidos por Lima-Camara cols ${ }^{17}$, ao passo que não se verificou associação entre a localização dos criadouros, no intradomicílio ou no peridomicílio, e a ocorrência de formas imaturas de Aedes aegypti. O fato de os depósitos serem ou não cobertos não influenciou a infestação por ambas as espécies, fato também observado por Bezerra ${ }^{18}$. A turbidez da água não constituiu um fator diferenciador quanto à presença de formas imaturas de Aedes albopictus, o que, provavelmente, revela um significativo potencial adaptativo deste frente ao Aedes aegypti, o qual apresenta predileção por locais que armazenam água límpida ${ }^{18}$. Apesar da progressiva e bem sucedida infestação do Aedes albopictus no ambiente urbano, onde há um predomínio por parte do Aedes aegypti, verificou-se que a ausência de uma espécie num criadouro parece facilitar a infestação pela outra (Tabela 1).

Pela análise da Tabela 2, observa-se que tanto o Aedes aegypti quanto o Aedes albopictus são capazes de colonizar criadouros dos mais variados volumes e altura. Contudo, quando comparados, deve-se ressaltar a preferência do Aedes albopictus por criadouros com volumes menores de água, assim como sua maior infestação em 
criadouros situados rente ao solo. Volumes maiores não apresentaram diferenças significativas em relação à infestação, quando comparados a volumes menores. Surteers ${ }^{19}$ descreve a influência dos volumes dos criadouros como determinantes para a infestação pelo Aedes aegypti, em que registra sua preferência por criadouros que concentram um maior volume de água. Na região de Mata Atlântica, bambus cortados que acumulavam água revelaram uma maior frequência de larvas de Aedes albopictus, em especial naqueles com volumes variando de 151 a 200ml, enquanto os negativos armazenavam menor volume de água ${ }^{20}$.

Analisando-se as alturas dos depósitos infestados pelo Aedes aegypti, no município de Fortaleza, Alencar ${ }^{21}$ relatou uma maior infestação daqueles situados numa altura média de 2,01m. Em estudo realizado no município de Quixadá, Estado do Ceará, no ano de 2005, foi observado uma altura média de $1,05 \mathrm{~m}$ para os criadouros infestados pelo Aedes aegypti ${ }^{22}$. Devido às características dos criadouros mais frequentados pelo Aedes albopictus, de acordo com Silva cols ${ }^{14}$, observou-se sua preferência por aqueles situados em alturas de aproximadamente $1 \mathrm{~m}$ do solo. Através dos resultados, percebeu-se que não houve diferença significativa quanto ao volume e à altura dos criadouros frente à infestação por ambas as espécies (Tabela 3).

Diante do potencial adaptativo de ambas as espécies em colonizar os mais variados criadouros no ambiente urbano, faz-se necessário expandir as estratégias de controle dos mosquitos vetores dos vírus dengue. Embora não se tenham casos confirmados na literatura acerca da transmissão dos vírus dengue por populações de Aedes albopictus, em episódios epidêmicos no país, não se pode descartar tal possibilidade, uma vez que cooperam para isso, as relações estabelecidas entre parasita e hospedeiro invertebrado, as quais são regidas, indubitavelmente, pelas variações genéticas existentes entre as cepas virais, bem como entre as populações desse culicídeo circulantes no território nacional ${ }^{23}$.

Conclui-se que as duas espécies se encontram dispersas por todo o Município de Fortaleza, ocupando os mais diversos tipos de criadouros, independentemente de seu volume ou altura. Observa-se uma maior frequência de Aedes albopictus no peridomicílio, enquanto Aedes aegypti ocupa indistintamente os ambientes intradomiciliar e peridomiciliar.

O Aedes albopictus, em virtude de seu ecletismo em frequentar os mais variados tipos de criadouros, exibe um potencial adaptativo bastante significativo quanto à colonização do espaço urbano de Fortaleza, já colonizado pelo Aedes aegypti.

\section{AGRADECIMENTOS}

À Secretaria Municipal de Saúde de Fortaleza pela parceria firmada no tocante à mobilização de recursos humanos para a coleta e o transporte das amostras utilizadas neste estudo.

\section{CONFLITO DE INTERESSE}

Os autores declaram não haver nenhum tipo de conflito de interesse.

\section{SUPORTE FINANCEIRO}

Ministério da Saúde (MS), Conselho Nacional de Desenvolvimento Científico e Tecnológico (CNPq) e Fundação Cearense de Amparo à Pesquisa (FUNCAP).

\section{REFERÊNCIAS}

1. Chiaravalloti-Neto F, Dibo MR, Barbosa AA, Battigaglia M.Aedes albopictus (S) na região de São José do Rio Preto, SP: estudo da sua infestação em área já ocupada pelo Aedes aegypti e discussão de seu papel como possível vetor de dengue e febre amarela. Rev Soc Bras Med Trop 2004; 35:351-357.

2. Lourenco-de-Oliveira R, Vazeille M, de Filippis AM, Failloux AB. Aedes aegypt in Brazil: genetically differentiated populations with high susceptibility to dengue and yellow fever viruses. Trans R Soc Trop Med Hyg 2004; 98:43-54.

3. Secretaria de Vigilância em Saúde. Programa Nacional de Controle da Dengue. Informe epidemiológico da dengue. Semanas de 1 a 30 de 2009, Ministério da Saúde, Brasília, 2009.

4. Alencar CHM, Braga LQV, Ramos JR AN, Lima JWO, Pontes RJS. Distribuição e Biologia do Aedes albopictus no Brasil: Um problema de Saúde Pública. J Bras Med 2008; 94:33-38.

5. Forattini OP. Identification of Aedes (Stegomyia) albopictus (Skuse) in Brazil Rev Saúde Pública 1986; 20:244-245.

6. Miller BR, Ballinger ME. Aedes albopictus mosquitoes introduced into Brazil vector competence for yellow fever and dengue viruses. Trans R Soc Trop Med Hyg 1988; 82:476-477.

7. Moore CG, Mitchell CJ. Aedes albopictus in the United States: ten-year presence and public health implications. Emerg Infect Dis. 1997; 3:329-334.

8. Martins VE, Martins MG, de Araujo JM, Silva LO, Monteiro HA, Castro FC et al. Primeiro registro de Aedes (Stegomyia) albopictus no Estado do Ceará, Brasil Rev Saúde Pública 2006; 40:737-739.

9. Marques GR, Forattini OP. Aedes albopictus em bromélias de solo em Ilhabela litoral do Estado de São Paulo. Rev Saúde Pública 2005; 39:548-552.

10. Instituto Brasileiro de Geografia e Estatística (IBGE). Acessado em 15 de Janeiro de 2009. Disponível em http://www.ibge.gov.br/home/estatistica/populacao/ contagem $2007 /$ default.shtm.

11. Consoli R, Lourenço RO. Principais mosquitos de importância sanitária no Brasil Rio de Janeiro: Fundação Instituto Oswaldo Cruz 1994.

12. Serufo JC, de Oca HM, Tavares VA, Souza AM, Rosa RV, Jamal MC et al. Isolation of dengue virus type 1 from larvae of Aedes albopictus in Campos Altos city, State of Minas Gerais, Brazil. Mem Inst Oswaldo Cruz 1993; 88:503-504.

13. Braks MA, Honorio NA, Lourenço-de-Oliveira R, Juliano SA, Lounibos LP. Convergent habitat segregation of Aedes aegypti and Aedes albopictus (Diptera: Culicidae) in southeastern Brazil and Florida. J Med Entomol 2003; 40:785-794.

14. Silva VC, Scherer PO, Falcão SS, Alencar J, Cunha J, Cunha SP, et al. Diversidade de criadouros e tipos de imóveis freqüentados por Aedes albopictus e Aedes aegypti. Rev Saúde Pública 2006; 40:1106-1111.

15. Forattini OP. Culicidologia Médica: identificação, biologia, epidemiologia. $1^{\text {a }}$ edição. Volume 2. São Paulo: Editora da Universidade de São Paulo; 2002.

16. Gomes AC, Forattini OP, Kakitani I, Marques GR, Marques CC, Marucci D, et al. Microhabitats de Aedes albopictus (Skuse) na região do Vale do Paraíba Estado de São Paulo, Brasil. Rev Saúde Pública 1992; 26:108-118.

17. Lima-Camara TN, Honorio NA, Lourenco-de-Oliveira R. Frequência e distribuição espacial de Aedes aegypti e Aedes albopictus (Diptera, Culicidae) no Rio de Janeiro, Brasil. Cad Saúde Pública 2006; 22:2079-2084.

18. Bezerra HSS. Determinantes da infestação domiciliar pelo Aedes aegypti na cidade de Fortaleza [Dissertação]. Fortaleza (CE): Universidade Federal do Ceará; 1999

19. Surteers G. Factors affecting the oviposition of Aedes aegypti. Bull Wld Hlth Org $1967 ; 36: 594-596$.

20. Silva AM, Nunes V, Lopes J. Culicídeos associados a entrenós de bambu bromélias, com ênfase em Aedes (Stegomyia) albopictus (Diptera, Culicidae) na Mata Atlântica, Paraná, Brasil. Iheringia, Ser Zool 2004; 94:63-66.

21. Alencar CHM. Infestação pelo Aedes albopictus (SKUSE), em criadouro naturais e artificiais encontrados em áreas verdes na cidade de Fortaleza-Ceará [Dissertação]. Fortaleza (CE): Universidade Federal do Ceará; 2008.

22. Alencar CHM. Determinantes da persistência de infestação pelo Aedes aegypti, em domicílios tratados com o temephos, no município de Quixadá [Especialização] Fortaleza (CE): Escola de Saúde Pública do Estado do Ceará; 2007.

23. Ayres CFJ, Romão TPA, Melo-Santos MAV, Furtado AF. Genetic diversity in brazilian populations of Aedes albopictus. Mem Inst Oswaldo Cruz 2002; 97:871-875. 